\title{
The Diabetes Excess Weight Loss (DEWL) Trial: a randomised controlled trial of high-protein versus high-carbohydrate diets over 2 years in type 2 diabetes
}

\author{
J. D. Krebs • C. R. Elley • A. Parry-Strong • H. Lunt • \\ P. L. Drury $\cdot$ D. A. Bell $\cdot$ E. Robinson $\cdot$ S. A. Moyes • \\ J. I. Mann
}

Received: 25 August 2011 / Accepted: 20 December 2011 /Published online: 28 January 2012

(C) Springer-Verlag 2012

\begin{abstract}
Aims/hypothesis To compare the effectiveness of low-fat high-protein and low-fat high-carbohydrate dietary advice on weight loss, using group-based interventions, among overweight people with type 2 diabetes.
\end{abstract}

Electronic supplementary material The online version of this article (doi:10.1007/s00125-012-2461-0) contains peer-reviewed but unedited supplementary material, which is available to authorised users.

J. D. Krebs · A. Parry-Strong · D. A. Bell

Department of Medicine, University of Otago,

Wellington, New Zealand

C. R. Elley $\cdot$ E. Robinson $\cdot$ S. A. Moyes

School of Population Health, University of Auckland,

Auckland, New Zealand

H. Lunt

Department of Medicine, University of Otago,

Christchurch, New Zealand

\section{P. L. Drury}

Auckland Diabetes Centre, Auckland District Health Board, Greenlane Clinical Centre,

Auckland, New Zealand

J. I. Mann

Edgar National Centre for Diabetes and Obesity Research (ENCDOR), Department of Medicine, Dunedin School of Medicine, University of Otago,

Dunedin, New Zealand

\section{Present address:}

J. D. Krebs $(\bowtie)$

Endocrine, Diabetes and Research Centre,

Capital and Coast Health,

Private Bag 7902,

Wellington, New Zealand

e-mail: jeremy.krebs@ccdhb.org.nz
Study design Multicentre parallel (1:1) design, blinded randomised controlled trial.

Methods Individuals with type 2 diabetes aged 30-75 years and a BMI $>27 \mathrm{~kg} / \mathrm{m}^{2}$ were randomised, by an independent statistician using sequentially numbered sealed envelopes, to be prescribed either a low-fat high-protein ( $30 \%$ of energy as protein, $40 \%$ as carbohydrate, $30 \%$ as fat) or a low-fat highcarbohydrate ( $15 \%$ of energy as protein, $55 \%$ as carbohydrate, $30 \%$ as fat) diet. Participants attended 18 group sessions over 12 months. Primary outcomes were change in weight and waist circumference assessed at baseline, 6 and 12 months. Secondary outcomes were body fatness, glycaemic control, lipid profile, blood pressure and renal function. A further assessment was undertaken 12 months after the intervention. Research assessors remained blinded to group allocation throughout. Intention-to-treat analysis was performed.

Results A total of 419 participants were enrolled (mean \pm SD age $58 \pm 9.5$ years, BMI $36.6 \pm 6.5 \mathrm{~kg} / \mathrm{m}^{2}$ and $\mathrm{HbA}_{1 \mathrm{c}} 8.1 \pm 1.2 \%$ $(65 \mathrm{mmol} / \mathrm{mol}))$. The study was completed by $70 \%(294 / 419)$. No differences between groups were found in change in weight or waist circumference during the intervention phase or the 12-month follow-up. Both groups had lost weight (2$3 \mathrm{~kg}, p<0.001)$ and reduced their waist circumference (2$3 \mathrm{~cm}, p<0.001$ ) by 12 months and largely maintained this weight loss for the following 12 months. By 6 months, the difference in self-reported dietary protein between groups was small $(1.1 \%$ total energy; $p<0.001)$. No significant differences between groups were found in secondary outcomes: body fatness, $\mathrm{HbA}_{1 \mathrm{c}}$, lipids, blood pressure and renal function. There were no important adverse effects.

Conclusions/interpretation In a 'real-world' setting, prescription of an energy-reduced low-fat diet, with either increased protein or carbohydrate, results in similar modest losses in weight and waist circumference over 2 years. 
Trial registration: Australia New Zealand Clinical Trials Register ACTRN12606000490572

Funding: The Health Research Council of New Zealand (06/337).

Keywords Diabetes mellitus type 2 .

Diet, carbohydrate-restricted $\cdot$ Randomised controlled trial .

Weight loss

$\begin{array}{ll}\text { Abbreviations } \\ \text { BP } & \text { Blood pressure } \\ \text { SF36 } & \text { 36-Item Short-Form Health Survey } \\ \text { UACR } & \text { Urinary albumin:creatinine ratio }\end{array}$

\section{Introduction}

Obesity is the primary modifiable risk factor for type 2 diabetes, and weight management is a major component of effective diabetes care. Dietary recommendations focus on an energy-reduced, low-fat diet with less than $10 \%$ of total energy derived from saturated fat and an emphasis on whole grains and foods with a low glycaemic index and high fibre $[1,2]$. However, individuals find these goals hard to achieve and even harder to maintain, with weight regain common [3].

Currently there is insufficient evidence to support one specific dietary approach for successful long-term weight loss, particularly in those with type 2 diabetes. This is reflected in the EASD guidelines and recent changes to the ADA recommendations [4]. However, these are based on relatively small studies or on meta-analyses of studies lasting 1 year or less. Substituting protein for carbohydrate while maintaining reduced total fat may have particular benefits in type 2 diabetes. High-protein diets promote weight loss, maintain lean body mass and improve lipid and glycaemic profiles in obese non-diabetic individuals [5-8]. Studies specifically in type 2 diabetes are limited, are generally short, often using very intensive interventions and/or providing a significant proportion of participants' food, making the translation of the findings to a general population difficult [9-11]. Therefore the specific long-term effect of increasing protein intake in individuals with type 2 diabetes in a free-living situation, using an intervention that can be realistically implemented, requires further investigation [12].

This multicentre randomised controlled trial assessed the effectiveness of prescribing a low-fat, high-protein dietary intervention compared with a low-fat, high-carbohydrate control diet in reducing weight and maintaining weight loss in overweight people with type 2 diabetes over a 2 -year period. It also assessed the effect of the diet on glycaemic control, lipid profile and blood pressure (BP) while monitoring adverse effects.

\section{Methods}

Study design The study was a multicentre parallel design, blinded randomised controlled trial.

Participants Participants were included if they had established type 2 diabetes (WHO criteria) [13], were between 30 and 76 years of age, and had a BMI of at least $27 \mathrm{~kg} / \mathrm{m}^{2}$.

Participants were excluded if they were currently on weight-reducing medications, had weight loss of $>5 \%$ in the past 3 months, or had a psychiatric or eating disorder. Participants were also excluded if their glycated haemoglobin $\left(\mathrm{HbA}_{1 \mathrm{c}}\right)$ was $>9.5 \%(80 \mathrm{mmol} / \mathrm{mol})$ or they had had renal disease (estimated glomerular filtration rate $<60 \mathrm{ml} / \mathrm{min}$ or urine albumin:creatinine ratio [UACR] $>30 \mathrm{mg} / \mathrm{mmol}$ ), abnormal liver enzymes, heart failure, known active malignancy or myocardial infarction in the preceding 6 months.

Recruitment Three centres in New Zealand (Wellington, Auckland and Christchurch) recruited participants during 2007 and 2008, using mail-out invitations through primary and secondary care to those with type 2 diabetes in the age range. Community and media advertisements were also used.

Randomisation and blinding Participants were randomised in a 1:1 ratio to either of two groups. One group was prescribed a low-fat, high-protein diet (40\% of total energy as carbohydrate, $30 \%$ as protein, $30 \%$ as fat) and the other, the active control group, a low-fat, high-carbohydrate diet (55\% of total energy as carbohydrate, $15 \%$ as protein, $30 \%$ as fat). Computer-generated randomisation was undertaken by an independent biostatistician. If two members from the same household were enrolled, they were both allocated to the same intervention group. Allocation of randomisation was concealed from the participant until after written informed consent and baseline assessment. Dieticians delivering the intervention were notified of the dietary allocation after baseline assessment and before the first group session; however, research assessors remained blind to intervention allocation at all assessment points and until after database lock.

Interventions Individuals participated in group sessions determined by dietary group allocation. Groups consisted of 812 participants and sessions were led by dieticians. The $1 \mathrm{~h}$ meetings were conducted every 2 weeks for the first 6 months, then every month for the second 6 months. The programmes were specifically designed for delivery in a 'real-world' setting, keeping the time commitment for both participants and staff to levels that could be readily achieved in most healthcare systems. There were no direct costs to participants to take part in this programme. The group sessions were identical for the two interventions, except the accompanying diet-specific information, and included 
an education component and time for discussion and concluded with goal setting. Dietary counselling included information on appropriate intakes of alcohol, saturated fat and fibre, the glycaemic index and behaviour change strategies. Weight was monitored at each session, and ongoing self-recording of food intake was encouraged. The same dieticians delivered sessions to both intervention groups to control for any differential effects of the individual dietician, and were trained by the investigating dietician (AP-S). Weekly text or email reminders and motivational messages were also offered to participants to enhance adherence to the diets.

The aim of both interventions was to reduce total energy intake by $2,000 \mathrm{~kJ} /$ day (approximately $500 \mathrm{kcal} /$ day) using an individualised dietary prescription based on estimation of energy requirements [1]. This was discussed on a one-to-one basis with each participant at the first group session. Portion charts were provided to enable a wide choice of everyday foods based on $15 \mathrm{~g}$ portions of protein and carbohydrate. Sample diet plans were also provided as a simpler option. Participants made their own food choices based on these guidelines. Culturally appropriate recipes were made available for specific ethnic groups. No further dietary advice was offered to either intervention group by the dieticians after 12 months. Participants were asked to continue following their prescribed diets on their own in the second year.

Outcome measures Outcome measures were assessed at baseline, 6, 12 and 24 months. Primary outcomes included weight (Tanita electronic scales (TBF300)) and waist circumference (smallest circumference between lower ribs and iliac crest). Secondary outcomes included glycaemic control $\left(\mathrm{HbA}_{1 \mathrm{c}}\right.$ [Roche Diagnostics, Mannheim, Germany] and fasting glucose), body fatness (bioimpedance), fasting lipid profile, systolic and diastolic BP, dietary adherence (3-day dietary diary), renal function (serum creatinine and UACR from $24 \mathrm{~h}$ urinary collection) and quality of life (36-Item Short-Form Health Survey [SF36]) [14]. Blood and urine samples were analysed in a centralised accredited laboratory. Renal function was also assessed by an unblinded data safety monitor 4 weeks after the start of the diets. All randomised participants were encouraged to return for assessment of end points regardless of dietary adherence.

Sample size Sample size calculations, using means and standard deviations from the literature and a previous pilot study, indicated that 420 participants would be required to detect clinically important differences between the groups of $1.9 \%$ in weight [15], $2 \mathrm{~cm}$ in waist circumference, and changes that have been achieved in other outcome variables from previous similar studies (see electronic supplementary material [ESM] Table 1) $(\alpha=80 \%, p=0.05)$. This sample size assumed missing data from $12 \%$ of participants at follow-up.
Analyses Intention-to-treat analyses were undertaken, regardless of adherence. Dietary adherence was estimated from the 3-day food diaries using the 2006 New Zealand Food Composition Database [16]. Generalised linear mixed models were used to investigate differences in changes over time in the two groups, including baseline as a covariate [17]. An increase, decrease or no change in BP-lowering and lipid-lowering medications was included in the model when relevant outcomes were being examined. Centre was included as a fixed effect in models. The mixed models used all available data, and missing data were assumed to be missing at random. Secondary analyses were undertaken examining change over time for each group.

Ethics Ethics approval was obtained from the Ministry of Health Multi-Regional Ethics Committee (MEC/06/08/081). Written informed consent was obtained from all participants.

\section{Results}

Of the 884 people who came forward, 419 (47\%) were eligible and enrolled (Fig. 1). Table 1 presents baseline characteristics of study participants; $70 \%$ (294/419) completed the 2-year assessment.

Table 2 presents the total energy and dietary macronutrient composition for both groups over the study. The high-protein group increased their protein intake significantly more than the high-carbohydrate group, but did not reach the target proportions. Both groups decreased total carbohydrate intake (g/day), with no significant difference between groups. However, as a percentage of total energy, carbohydrate intake remained high and became significantly higher in the highcarbohydrate group. Both groups decreased their total energy intake, with the high-carbohydrate group reporting significantly lower intake than the high-protein group. However, there was no significant difference between the groups in change in weight or waist circumference over the 2 years (Table 3), although these measures decreased significantly from baseline in both groups, particularly in the first 6 months (Fig. 2). There was no difference in the change in percentage body fat and $\mathrm{HbA}_{1 \mathrm{c}}$ between the groups (Table 3). However, compared with baseline, there were significant reductions in percentage body fat and $\mathrm{HbA}_{1 \mathrm{c}}$ in both groups in the first 6 months ( $p \leq 0.01$; ESM Fig. 1).

There were no significant differences between the groups in any of the secondary outcomes, except total cholesterol and triacylglycerols, where the change over time followed a different pattern, but the final results at 24 months were similar. Total cholesterol had decreased in both groups at 24 months compared with baseline $(0.24 \mathrm{mmol} / 1$ in the highprotein group and $0.17 \mathrm{mmol} / 1$ in the high-carbohydrate group), but there was no difference between groups in 


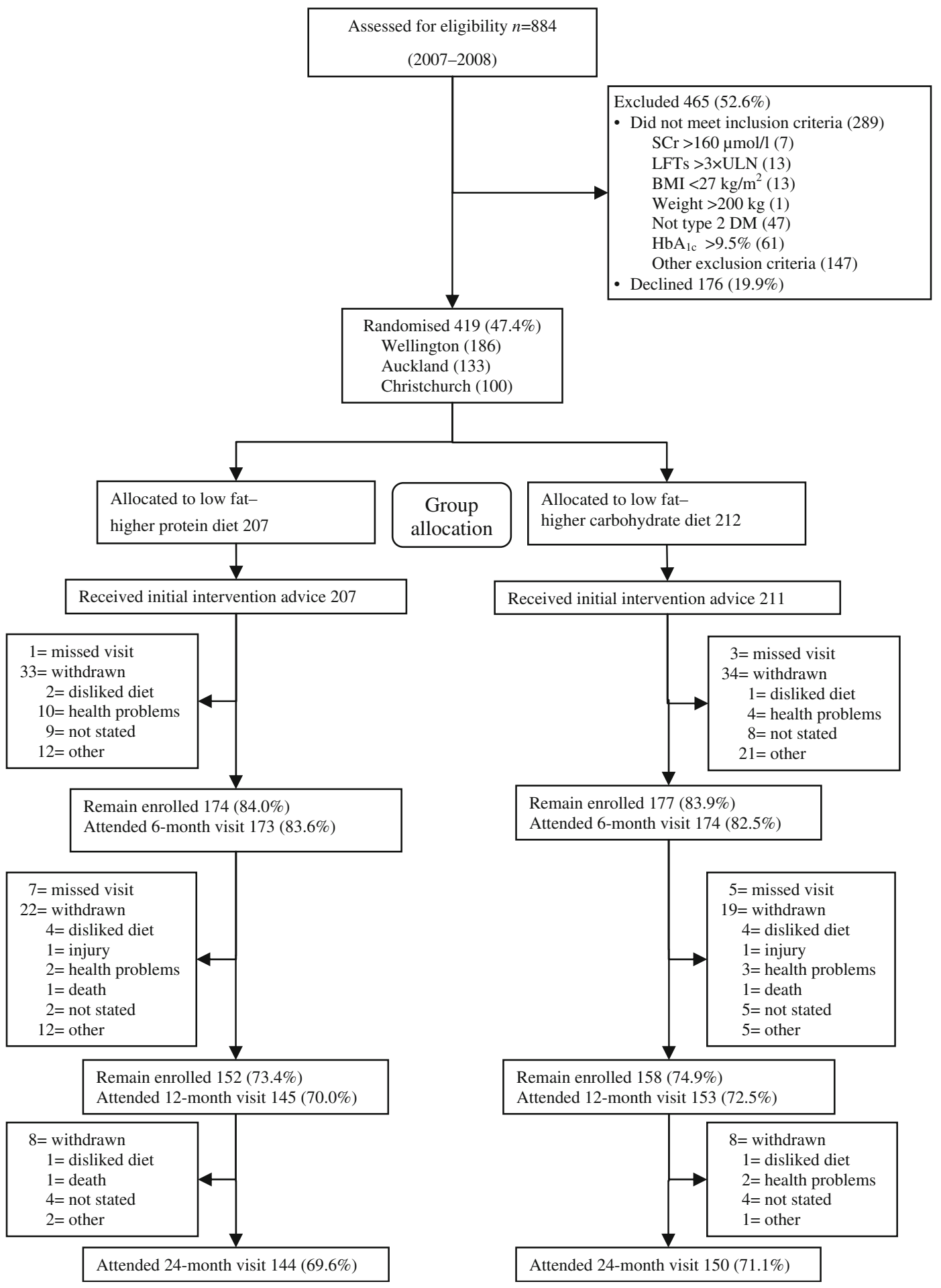

Fig. 1 Flow diagram for the Diabetes Excess Weight Loss (DEWL) Trial: high-protein vs high-carbohydrate dietary interventions

absolute change. Triacylglycerol levels decreased more in the high-protein group between baseline and 12 months, but both groups had returned to baseline levels by 24 months (Table 3).

There was no difference in the number of adverse renal events between the groups. Serum creatinine had increased by $\geq 15 \mu \mathrm{mol} / 1$ from baseline at any of the follow-up measurements in $32(18.5 \%)$ of the participants in the high-protein group compared with $36(20.7 \%)$ in the high-carbohydrate group ( $p=0.6$; ESM Table 2).

The mean age of those who dropped out of trial assessments was higher than those completing the trial (59 vs 
Table 1 Baseline characteristics of trial participants by group allocation

\begin{tabular}{|c|c|c|c|}
\hline Characteristic & $\begin{array}{l}\text { High } \\
\text { protein }\end{array}$ & $\begin{array}{l}\text { High } \\
\text { carbohydrate }\end{array}$ & Total \\
\hline Number & 207 & 212 & 419 \\
\hline Age (years) & $57.7 \pm 9.9$ & $58.0 \pm 9.2$ & $57.9 \pm 9.5$ \\
\hline Male & $95(46)$ & $73(34)$ & $168(40)$ \\
\hline \multicolumn{4}{|l|}{ Ethnicity } \\
\hline European & $167(81)$ & $185(88)$ & $352(84)$ \\
\hline Maori & $22(11)$ & $19(9)$ & $41(10)$ \\
\hline Pacific & $8(4)$ & $6(3)$ & $14(3)$ \\
\hline Other & $14(7)$ & $7(3)$ & $21(5)$ \\
\hline Duration of diabetes (years) & $8.3 \pm 6.6$ & $8.2 \pm 6.3$ & $8.2 \pm 6.5$ \\
\hline BMI $\left(\mathrm{kg} / \mathrm{m}^{2}\right)$ & $36.6 \pm 6.7$ & $36.7 \pm 6.4$ & $36.6 \pm 6.5$ \\
\hline $\mathrm{HbA}_{1 \mathrm{c}}(\%)$ & $8.1 \pm 1.2$ & $8.0 \pm 1.2$ & $8.1 \pm 1.2$ \\
\hline $\mathrm{HbA}_{1 \mathrm{c}}(\mathrm{mmol} / \mathrm{mol})$ & $65 \pm 11$ & $64 \pm 11$ & $65 \pm 11$ \\
\hline Microalbuminuria or greater ${ }^{\mathrm{a}}$ & $37(18.0)$ & $40(19.0)$ & $77(18.5)$ \\
\hline \multicolumn{4}{|c|}{ Glycaemic control } \\
\hline Diet only & $40(19.3)$ & $29(13.9)$ & $69(16.6)$ \\
\hline Oral agents only & $116(56.0)$ & $120(57.4)$ & $236(56.7)$ \\
\hline Insulin \pm oral agents & $51(24.6)$ & $60(28.7)$ & $111(26.7)$ \\
\hline On lipid-lowering drugs & $128(61.8)$ & $147(69.3)$ & $275(65.6)$ \\
\hline On BP-lowering drugs & $160(77.3)$ & $158(74.5)$ & $318(75.9)$ \\
\hline
\end{tabular}

Values are mean \pm SD or $n(\%)$

${ }^{\text {a }} \mathrm{UACR} \geq 2.5 \mathrm{mg} / \mathrm{mmol}$ in men and $\geq 3.5 \mathrm{mg} / \mathrm{mmol}$ in women

55 years, $p<0.001)$. They also had higher $\mathrm{HbA}_{1 \mathrm{c}}$ at baseline $(8.2 \%$ vs $8.0 \%$ [66 vs $64 \mathrm{mmol} / \mathrm{mol}], p=0.04)$, but the difference in their weight was not statistically significant (105 vs $102 \mathrm{~kg}, p=0.14)$. Complete case analyses, including only those who completed the trial, still found no significant difference between the groups in primary outcomes. A per protocol analysis was undertaken including only those in each group that achieved above the median intake at 6 months of protein $(89 \mathrm{~g} /$ day $)$ or carbohydrate $(192.5 \mathrm{~g} /$ day $)$ in the intervention and control groups, respectively. There was no significant difference at any time point or overall between the groups in the three variables examined, including weight $(p=0.8)$, waist circumference $(p=0.7)$ and $\operatorname{HbA}_{1 \mathrm{c}}(p=0.9)$ (ESM Table 3).

\section{Discussion}

The main finding of this randomised controlled dietary intervention trial was that, in overweight and obese individuals with type 2 diabetes, the prescription of a high-protein, carbohydrate-restricted diet does not promote greater weight loss or reduction in waist circumference than a low-fat highcarbohydrate diet over 2 years. A key finding was the modest weight loss achieved with realistic and sustainable support in a community setting, regardless of advice about dietary content. Overall weight loss was similar in the two groups. The result supports recommendations for flexibility with macronutrient compositions for weight management in those with diabetes, but reinforces the importance of achieving sustained reductions in total energy intake.

Strengths of this study include the careful randomised controlled design, the large number of participants and the 2 year length of follow-up compared with other similar dietary intervention studies, the multicentre recruitment and the focus on an intervention, which can be realistically achieved in real world everyday practice. Limitations include the relatively high drop out rate, the failure of individuals to achieve and maintain target diet macronutrient compositions, and the lack of robust biomarkers of dietary adherence. This is particularly important in terms of achieving differences between groups in total amounts of carbohydrate and protein. The study was powered with a sample size calculation that assumed that $12 \%$ of the data would be missing at follow-up, yet $30 \%$ of the data were missing at 24 months. The attrition rate was similar in the two groups, but those who dropped out tended to be older and to have a higher $\mathrm{HbA}_{1 \mathrm{c}}$ at baseline, although their weight was not significantly different from those who completed the study. Both the 'intention-to-treat' analysis and the analysis that only included those who completed the study found no difference between the groups in any outcome. There is the potential that a real difference between groups has been missed. However, the number of participants is still considerably larger than in any comparable studies, and, given the small effect sizes and the consistency across the outcomes observed, this would seem unlikely. Another potential limitation was the multiple outcomes measured, which may have risked a type 1 error. However, no significant difference was found in any outcome.

There has been considerable interest in the role of manipulating dietary macronutrient composition as a mechanism for achieving weight loss. Recent focus on 'low-carb' diets such as the 'Dr Atkins diet revolution' [18] and 'The Zone' diet [19] has some supportive evidence for short-term weight loss over 6 months, but no difference compared with other diets at 12 months $[15,20-23]$. In the present study, total self-reported carbohydrate intake in grams per day actually decreased in both groups, but could not be considered 'low carbohydrate' and should not be compared with other specific low-carbohydrate diets. However, as a proportion of total energy intake, carbohydrate remained above $40 \%$ in both groups and, as intended, was significantly greater in the high-carbohydrate group.

Large reductions in dietary carbohydrate are by necessity paralleled by major increases in the proportion, and often actual levels, of fat and protein intake. This is particularly concerning if the intake of saturated fat increases. Evidence 


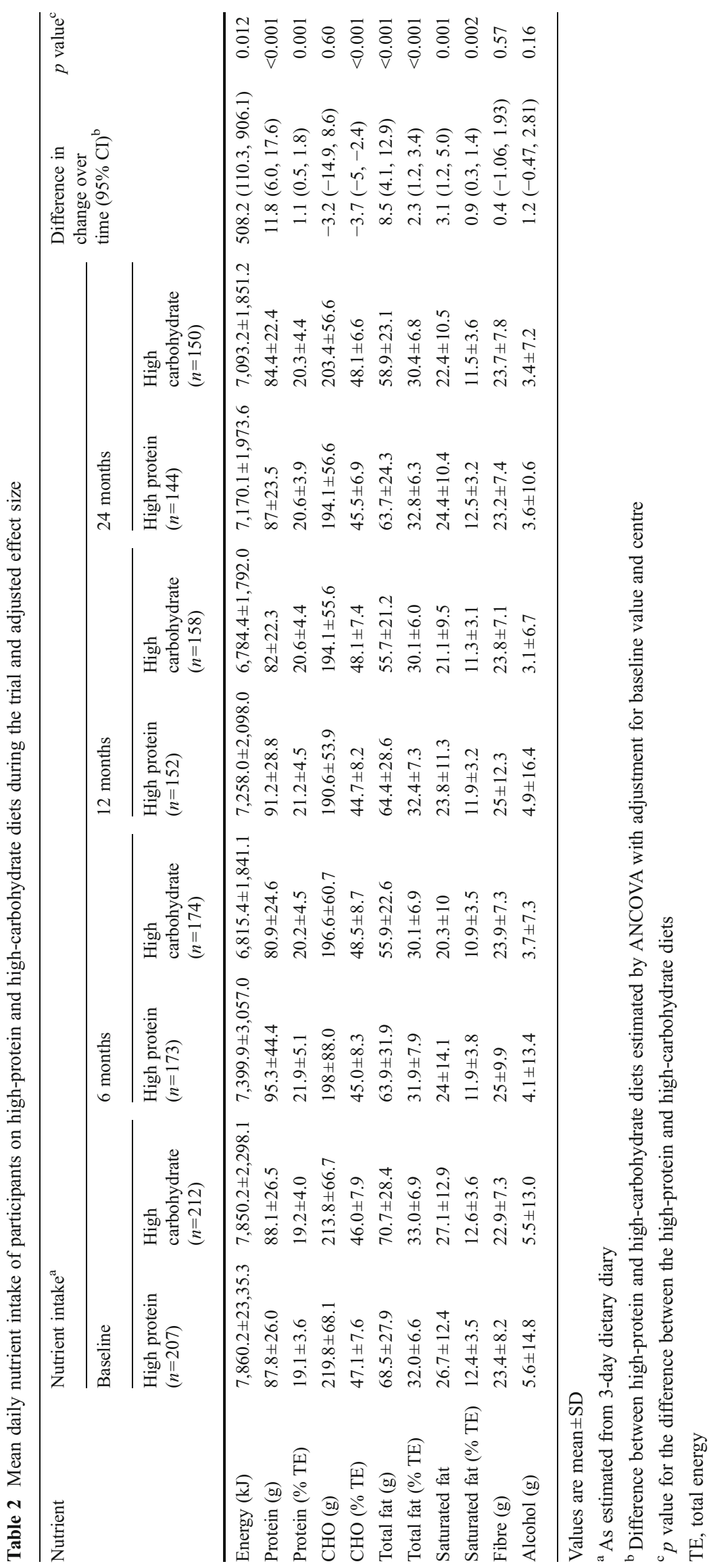




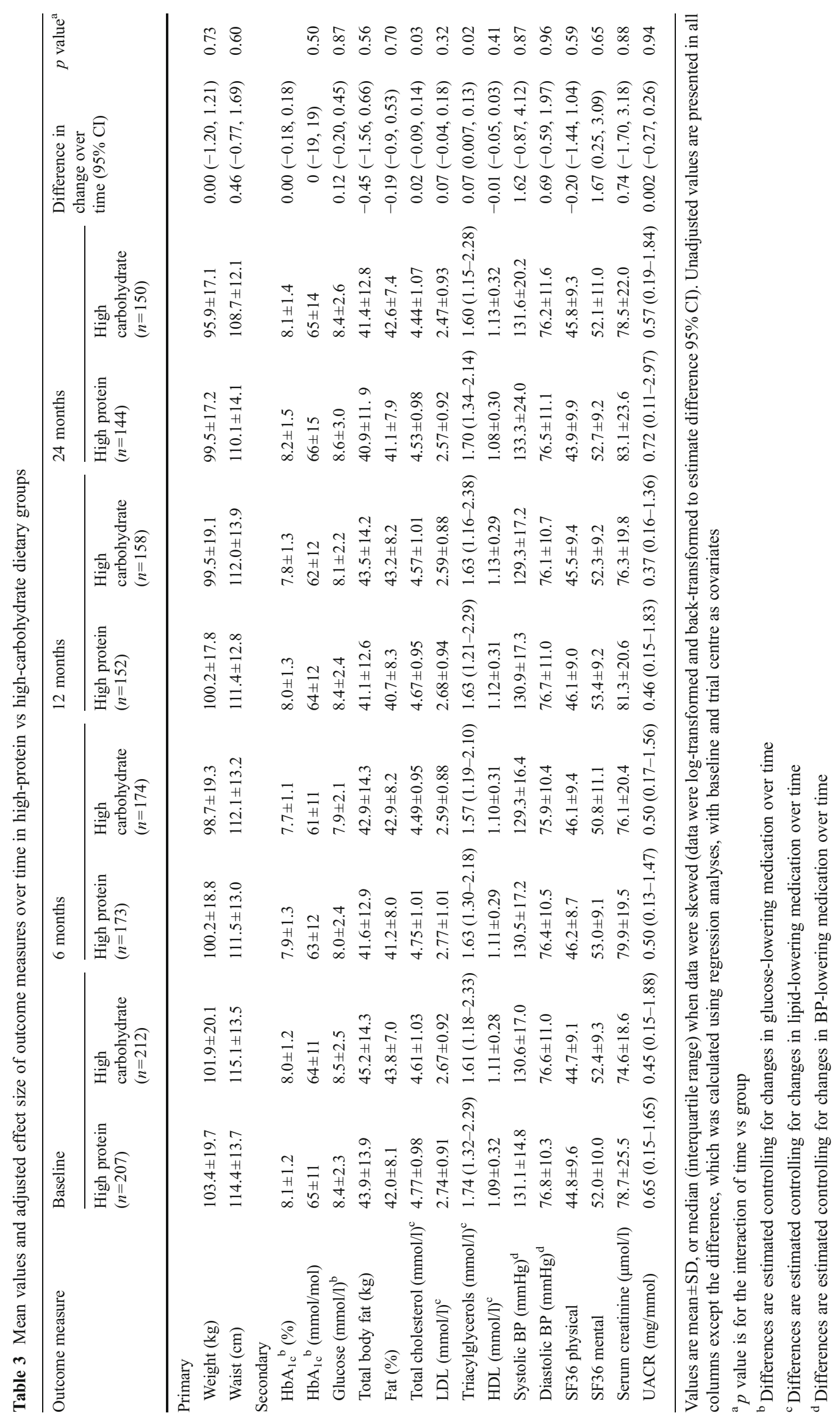




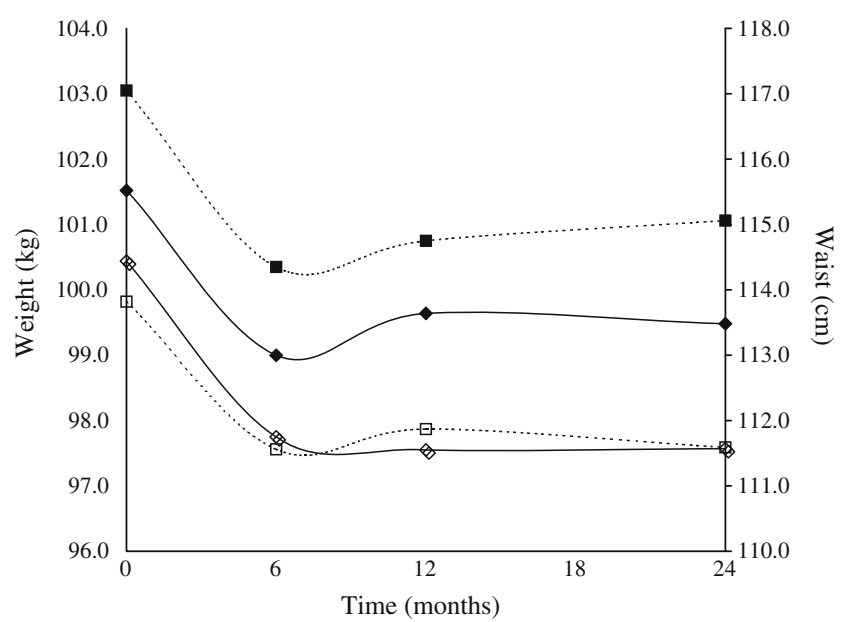

Fig. 2 Change in weight and waist over time in the intervention (highprotein) and control (high-carbohydrate) groups, estimated from generalised linear mixed models. Black diamond, control weight; black square, intervention weight; white diamond, control waist; white square, intervention waist. Weight and waist circumference had decreased significantly $(p<0.001)$ in the intervention and control groups when baseline was compared with 6-month measures and when baseline was compared with the mean of 6,12 and 24 months

supports substitution with protein, monounsaturated or polyunsaturated fats. The prescribed intervention diet in this study recommended replacing carbohydrate with protein. By adopting more moderate changes than the extreme popular diets, it was proposed that adherence, the critical component of successful weight loss, would be enhanced [24]. However, in this study, as in previous dietary intervention studies, the drop out rate was high in both groups, with 'difficulty adhering' to either diet cited by participants as a major factor.

An interesting finding was that, while prescribed protein in the high-protein diet was $30 \%$, this target was achieved in only 12 of the 207 (6\%) participants in the high-protein group. Protein intake had significantly increased (mean $+8 \mathrm{~g}$ per day) at 6 months in the high-protein group, but, at $21.9 \%$ of total energy, was less than the $25 \%$ level considered to be 'high protein'. Furthermore, in both dietary groups, the macronutrient composition trended back to baseline proportions between 6 months and 2 years, indicating a strong drive to habitual dietary patterns and emphasising the difficulty in making sustained changes to these.

Self-reported food diaries have well-recognised limitations, and under-reporting of energy intake is common. This may have confounded interpretation of intake data, as the mean total reported energy intake in the control diet was significantly lower than in the high-protein diet, yet this did not translate to greater weight loss. Furthermore, without supplying participants with food, we cannot be certain that food diary reports accurately reflect the intake over the duration of the study. This limitation also precludes further analysis regarding the extent to which reported amounts of carbohydrate influence outcome measures. It is generally accepted that food diaries provide a more reliable measure of relative contribution to dietary energy than absolute amounts of individual macronutrients $[25,26]$. The use of $24 \mathrm{~h}$ urinary nitrogen measurements as a biomarker of protein intake may have been a valuable addition to this study. However, the approach of prescribing a diet with supportive material and group sessions is normal practice in daily primary and secondary care and as such was an integral component in the design of this study.

Previous trials where weight loss and changes in insulin sensitivity, glycaemic control, lipids and BP were greater with high-protein than high-carbohydrate diets were generally shorter, conducted in non-diabetic groups, with more intensive interventions or with provision of supplements or food. Brinkworth et al [27] supplied $60 \%$ of participants' food intake, achieving a protein intake of $28 \%$, but, once the supply of food was stopped during the follow-up period, protein intake fell in the high-protein group to just $21 \%$ after 3 months. Larsen et al [28] recently reported a 12-month intervention trial in a similar population of patients with type 2 diabetes, with similar dietary goals to the present study, comparing a high-protein and high-carbohydrate diet. They were able to achieve a greater difference between groups in actual protein intake, but were also unable to reach their goal composition. This was despite considerably greater one-on-one intervention from a dietician ( $5 \mathrm{~h}$ per person over 12 months) and additional group sessions. Larsen et al concluded that over 12 months there was no difference between dietary approaches for $\mathrm{HbA}_{1 \mathrm{c}}$, weight or other secondary outcomes. The present study was specifically designed to test whether prescription of a highprotein diet with support in a manner that could be directly translated to real-world practice with limited resources could achieve similar results to previous short-term, highly controlled trials over the long term. It is clear that the level of intervention adopted in this trial was not sufficient to facilitate comprehensive or sustained dietary change, as the target macronutrient compositions were not achieved by either group, and the differences in composition decreased over time.

Concern has been raised that increased protein intake, particularly in those with microalbuminuria or established diabetic nephropathy, could cause a deterioration in renal function. In this study with modest increases in protein intake, there was no change in microalbuminuria in either group, and no individuals showed any clinically significant rise in creatinine.

While we cannot conclude with certainty that a high-protein diet is not superior to a high-carbohydrate diet for weight loss in those with type 2 diabetes, the results of this study combined with those of Larsen et al [28] indicate that, with interventions realistically deliverable in a real-world setting, a focus on macronutrient composition is not an effective strategy for weight loss. Future research should focus on reducing the barriers to sustained behavioural change to achieve reductions 
in energy intake in free-living individuals. Highly controlled dietary studies are unlikely to answer this challenge. As the drop outs in this study were more likely to be older and have a higher starting $\mathrm{HbA}_{1 \mathrm{c}}$, future research should also look at ways of better engaging this target population.

In conclusion, this large community-based randomised controlled dietary intervention study has shown that, in patients with type 2 diabetes, prescription of a high-protein diet with a group-based dietician-led support and education programme easily deliverable in a real-world setting does not promote greater weight loss than the prescription of a highcarbohydrate diet. Both diets promoted modest and sustained weight loss over 2 years. This adds further evidence to the growing understanding that dietary prescriptions focusing on macronutrient composition is not an effective strategy in achieving meaningful weight loss in free-living overweight and obese individuals. It again highlights how difficult it is to achieve and maintain any prescribed change in dietary composition, and how individuals trend back to habitual intakes over time. Furthermore, this study does not support the notion that high-protein intakes have any greater benefit on glycaemic control, lipid profile or BP, nor does there appear to be any detrimental effect on renal function. Therefore this study reinforces the need to find ways to achieve sustained reduction in total energy intake as the primary focus to achieve longterm weight loss and supports a flexible approach to dietary composition for individuals with type 2 diabetes.

Acknowledgements We would like to acknowledge the participants of the trial, who have contributed their effort and time to the trial. We also thank the research nurses, in particular C. Ross, C. Patten, F. Logan, L. Shaw, D. Walker, T. Clarke and I. McEnaney, and the research assistants M. Jefferies, C. Maister and H. Hantz. We are also grateful to the study dieticians F. McKechnie, C. Cairncross, G. Dunkley, K. Corselius White and S. Benny, who delivered the dietary interventions. We further acknowledge A. Duncan and the Diabetes \& Lipid Laboratory, Department of Human Nutrition of the University of Otago for analysing all blood and urine samples.

Funding This trial was funded by the Health Research Council of New Zealand (06/337).

Duality of interest The authors declare that there is no duality of interest associated with this manuscript.

Contribution statement All authors contributed to the study design and writing and revision of the manuscript and approved the final version. JK was the principal investigator of the study, developed the intervention, and led the writing of the manuscript. JK also led the Wellington trial centre. CRE was a co-principal investigator and led the trial design. AP-S developed the intervention protocols and oversaw the delivery of the interventions. HL and PLD led the trial at the two trial sub-centres, Christchurch and Auckland. DB also led the trial in Wellington and oversaw metabolic assessment. ER was the trial biostatistician and undertook the main analyses. SM was the data manager and contributed to analyses. JM advised on intervention, development and interpretation of results.

\section{References}

1. Franz MJ, Bantle JP, Beebe CA et al (2004) Nutrition principles and recommendations in diabetes. Diabetes Care 27(Suppl 1):S36S46

2. Mann JI, de Leeuw I, Hermansen K et al (2004) Evidence-based nutritional approaches to the treatment and prevention of diabetes mellitus. Nutr Metab Cardiovasc Dis 14:373-394

3. Wadden TA, Foster GD (2000) Behavioral treatment of obesity. Med Clin N Am 84:441-461

4. American Diabetes Association (2008). Nutrition Recommendations and Interventions for Diabetes. Diabetes Care 31(Suppl 1): S61-S78.

5. Layman DK, Evans E, Baum JI et al (2005) Dietary protein and exercise have additive effects on body composition during weight loss in adult women. J Nutr 135:1903-1910

6. Claessens M, van Baak MA, Monsheimer S, Saris WH (2009) The effect of a low-fat, high-protein or high-carbohydrate ad libitum diet on weight loss maintenance and metabolic risk factors. Int $\mathrm{J}$ Obes 33:296-304

7. Skov AR, Toubro S, Ronn B, Holm L, Astrup A (1999) Randomized trial on protein vs carbohydrate in ad libitum fat reduced diet for the treatment of obesity. Int J Obes 23:528-536

8. Due A, Toubro S, Skov AR et al (2004) Effect of normal-fat diets, either medium or high in protein, on body weight in overweight subjects: a randomised 1-year trial. Int J Obes 28:1283-1290

9. Gannon MC, Nuttall FQ, Gannon MC, Nuttall FQ (2004) Effect of a high-protein, low-carbohydrate diet on blood glucose control in people with type 2 diabetes. Diabetes 53:2375-2382

10. Sargrad KR, Homko C, Mozzoli M et al (2005) Effect of high protein vs high carbohydrate intake on insulin sensitivity, body weight, hemoglobin A1c, and blood pressure in patients with type 2 diabetes mellitus. JADA 105:573-580

11. Evangelista LS, Heber D, Li Z et al (2009) Reduced body weight and adiposity with a high-protein diet improves functional status, lipid profiles, glycemic control, and quality of life in patients with heart failure: a feasibility study. J Cardiovasc Nurs 24:207-215

12. Layman DK, Clifton P, Gannon MC, Krauss RM, Nuttall FQ (2008) Protein in optimal health: heart disease and type 2 diabetes. Am J Clin Nutr 87:1571S-1575S

13. Alberti KG, Zimmet PZ (1998) Definition, diagnosis and classification of diabetes mellitus and its complications. Part 1: diagnosis and classification of diabetes mellitus provisional report of a WHO consultation. Diabet Med 15:539-553

14. McHorney CA, Ware JE Jr, Raczek AE (1993) The MOS 36-Item Short-Form Health Survey (SF-36): II. Psychometric and clinical tests of validity in measuring physical and mental health constructs. Medical Care 31:247-263

15. Foster GD, Wyatt HR, Hill JO et al (2003) A randomized trial of a low-carbohydrate diet for obesity. N Engl J Med 348:2082-2090

16. New Zealand Crop and Food Research (2006) FOODfiles. New Zealand Food Composition Database (CD). New Zealand Crop and Food Research, Palmerston North, New Zealand

17. Diggle P (2002) Analysis of longitudinal data. 2nd edn: Oxford University Press

18. Atkins R (1992) Dr Atkins' new diet revolution. Avon Books Inc, New York

19. Sears B (2000) A week in the Zone, 1st edn. Harper Collins, New York

20. Bravata DM, Sanders L, Huang J, Krumholz HM, Olkin I, Gardner CD (2003) Efficacy and safety of low-carbohydrate diets: a systematic review. Jama 9(289):1837-50

21. Brehm BJ, Seeley RJ, Daniels SR, D'Alessio DA (2003) A randomized trial comparing a very low carbohydrate diet and a calorierestricted low fat diet on body weight and cardiovascular risk factors in healthy women. J Clin Endocrinol Metab 88:1617-1623 
22. McAuley KA, Hopkins CM, Smith KJ et al (2005) Comparison of high-fat and high-protein diets with a high-carbohydrate diet in insulin-resistant obese women. Diabetologia 48:8-16

23. Samaha FF, Iqbal N, Seshadri P et al (2003) A low-carbohydrate as compared with a low-fat diet in severe obesity. N Engl J Med 348:2074-2081

24. Sacks FM, Bray GA, Carey VJ et al (2009) Comparison of weightloss diets with different compositions of fat, protein, and carbohydrates. N Engl J Med 360:859-873

25. Cook A, Pryer J, Shetty P (2000) The problem of accuracy in dietary surveys. Analysis of the over 65 UK National Diet and Nutrition Survey. J Epidemiol Commun Health 54:611-616
26. Hoidrup S, Andreasen AH, Osler M et al (2002) Assessment of habitual energy and macronutrient intake in adults: comparison of a seven day food record with a dietary history interview. Eur J Clin Nutr 56:105-113

27. Brinkworth GD, Noakes M, Keogh JB, Luscombe ND, Wittert GA, Clifton PM (2004) Long-term effects of a high-protein, lowcarbohydrate diet on weight control and cardiovascular risk markers in obese hyperinsulinemic subjects. Int J Obes 28:661-670

28. Larsen RN, Mann NJ, Maclean E, Shaw JE (2011) The effect of high-protein, low-carbohydrate diets in the treatment of type 2 diabetes: a 12 month randomised controlled trial. Diabetologia 20:20 